\title{
PRÁTICA EDUCATIVA COM ENFERMEIROS DA ATENÇÃO PRIMÁRIA: NÃO À LESÃO POR PRESSÃO*
}

\author{
Cilene Fernandes Soares' ${ }^{1}$ Ivonete Teresinha Shulter Buss Heidemann², Michelle Kuntz Durand ${ }^{3}$, \\ Maria Fernanda Baeta Neves Alonso da Costa ${ }^{4}$, Claudia Cossentino Bruck Marçal' \\ Juliana Martins Ferreira ${ }^{6}$
}

\begin{abstract}
Objetivos: Analisar, a partir da visão dos enfermeiros da Atenção Primária, as contribuições da prática educativa na prevenção da Lesão por Pressão e promoção da saúde. Método: Estudo Convergente Assistencial com 20 enfermeiros, realizado entre julho e agosto de 2014, no Estado de Santa Catarina, desenvolvido em duas etapas e quatro encontros, sendo a primeira entrevista com a utilização de questionário e, posteriormente, três oficinas. Resultados: Os dados foram analisados e resultaram em duas categorias: Diga não à Lesão por Pressão e A prática do enfermeiro na prevenção da LP e promoção da saúde na APS, que expressaram aquisição e aprimoramento de conhecimentos, construção do novo saber, contribuindo para ações de saúde. Conclusão: A prática educativa alcançou seu propósito, inovando e renovando o conhecimento dos enfermeiros no cuidado preventivo e promoção da saúde. São essenciais capacitações contínuas, refletindo em ganhos na prática assistencial e científica da Enfermagem.
\end{abstract}

DESCRITORES: Enfermagem; Lesão por pressão; Atenção primária à saúde; Prevenção de doenças; Promoção da saúde.

\section{EDUCATIONAL PRACTICE WITH PRIMARY CARE NURSES: SAY NO TO PRESSURE ULCER}

Objectives: To analyze, from the views of Primary Care nurses, the contributions of educational practice in the prevention of Pressure Ulcer and health promotion. Method:. Convergent Care research with 20 nurses conducted between July and August 2014, in the State of Santa Catarina, developed in two stages and including four meetings. First, the nurses were interviewed, through the use of a questionnaire, and subsequently participated in three workshops. Results: Data was submitted to analysis, and two categories emerged: Say no to Pressure Ulcer and The practices developed by nurses in the prevention of $\mathrm{PU}$ and health promotion in PHC, which expressed acquisition and improvement of knowledge, construction of new knowledge, contributing to the promotion of health actions. Conclusion: The educational practice was successful, as it promoted innovation and the nurses gained more knowledge on preventive health care and health promotion. Continuous training is necessary, as it improves nursing care and scientific practice. KEYWORDS: Nursing; Pressure ulcer; Primary health care; Prevention of diseases; Health promotion.

\section{PRÁCTICA EDUCATIVA CON ENFERMEROS DE ATENCIÓN PRIMARIA: NO A LA LESIÓN POR PRESIÓN}

Objetivos: Analizar, partiendo de la visión de los enfermeros de Atención Primaria, las contribuciones de la práctica educativa en la prevención de la Lesión por Presión y promoción de salud. Método: Estudio Convergente Asistencia, con 20 enfermeros, realizado entre julio y agosto de 2014 en Santa Catarina, desarrollado en dos etapas y cuatro reuniones, utilizándose cuestionario en la primera entrevista, realizándose luego tres talleres. Resultados: Datos analizados resultando en dos categorías: Dígale No a la Lesión por Presión y La práctica del enfermero en la prevención de la LP y la promoción de la salud en la APS, expresando adquisición y mejoramiento de conocimientos, construcción de nuevo saber, contribuyendo a acciones sanitarias. Conclusión: La práctica educativa consiguió su propósito, innovando y renovando el conocimiento de los enfermeros en el cuidado preventivo y promoción de salud. Resultan esenciales capacitaciones continuas, reflexionando sobre avances en la práctica asistencial y científica de Enfermería.

DESCRIPTORES: Enfermería; Úlcera por Presión; Atención Primaria de Salud; Prevención de Enfermedades; Promoción de la Salud.

\footnotetext{
* Artigo extraído da dissertação intitulada: Úlcera por Pressão no contexto da Atenção Primária: reflexão com enfermeiros sobre a escala de Braden. Programa de Pós-Graduação em Enfermagem da Universidade Federal de Santa Catarina, 2015.

'Enfermeira. Mestre em Enfermagem pelo Programa de Pós-Graduação em Enfermagem da Universidade Federal de Santa Catarina, Enfermeira Assistencial Secretaria Municipal de Saúde de Florianópolis. Florianópolis, SC, Brasil.

²Enfermeira. Doutora em Enfermagem em Saúde Pública, Professora Associada do Departamento de Enfermagem, Programa de PósGraduação em Enfermagem da Universidade Federal de Santa Catarina. Florianópolis, SC, Brasil.

${ }^{3}$ Enfermeira. Doutora em Enfermagem pelo Programa de Pós-Graduação em Enfermagem da Universidade Federal de Santa Catarina, Chapecó, SC, Brasil. Docente Colaboradora do Departamento de Enfermagem da Universidade do Estado de Santa Catarina UDESC/ CEO. Florianópolis, SC, Brasil.

${ }^{4}$ Enfermeira. Doutora em Enfermagem em Saúde Coletiva, Professora Adjunta do Departamento de Enfermagem do Departamento de Enfermagem da Universidade Federal de Santa Catarina, Florianópolis, SC, Brasil.

${ }^{5}$ Fonoaudióloga. Doutoranda do Programa de Pós-Graduação em Enfermagem da Universidade Federal de Santa Catarina, Florianópolis, SC, Brasil.

${ }^{6}$ Enfermeira. Mestre em Enfermagem pelo Programa de Pós-Graduação em Enfermagem da Universidade Federal de Santa Catarina. Florianópolis, SC, Brasil.
} 


\section{INTRODUÇÃO}

O cuidado de Enfermagem pode ser compreendido como um processo que envolve e desenvolve ações, atitudes e comportamentos que se fundamentam no conhecimento científico, técnico, cultural, social, econômico, político e psicoespiritual, buscando a promoção, manutenção e ou recuperação da saúde e dignidade humana. ${ }^{(1)}$

A Enfermagem tem na prática educativa uma de suas linhas norteadoras, essencial para formação e atualização, inclusive dos cuidados preventivos e de promoção da saúde. ${ }^{(2)}$ Essa prática admite o debate, a reflexão e os resultados expressivos por considerar os sujeitos ativamente participantes no processo de ensino e aprendizagem, ${ }^{(3)}$ caracterizando-se como um potencializador na forma de prestar o cuidado. ${ }^{(4)}$

Entendido como alicerce, o cuidado fundamenta a prática da Enfermagem. À luz desse pensamento, associam-se os cuidados com a integridade da pele com vistas à promoção da saúde e prevenção de doenças, cuja atenção pode levar a menor índice de desenvolvimento da Lesão por Pressão (LP).

A LP é uma lesão tissular, localizada geralmente sobre uma proeminência óssea ou relacionada ao uso de um dispositivo médico ou outro artefato, em resultado da pressão intensa e/ou prolongada em combinação com o cisalhamento. ${ }^{(5)}$

No cuidado de Enfermagem preventivo, as ferramentas de avaliação de risco para o desenvolvimento da Lesão por Pressão direcionam a assistência. Dentre essas, a escala de Braden tem por finalidade identificar as pessoas vulneráveis. Esta é composta por seis subescala que refletem a percepção sensorial, a umidade, o estado nutricional, a atividade e a mobilidade, fricção e cisalhamento. A somatória dos escores resulta em valores entre seis e 23, e quanto menor a pontuação maior o risco de desenvolver o agravo. ${ }^{(6)}$

Nesse contexto, a avaliação de risco para LP está ganhando espaço no cotidiano assistencial dos profissionais da saúde em virtude das consequências de uma lesão, tais como restrição de mobilidade e atividade, expansão da necessidade de internações hospitalares, aumento do risco de infecção e sepse, além do elevado custo para o tratamento de tais consequências. ${ }^{(7-8)}$

Na Europa, um estudo realizado para explorar o estado atual de desenvolvimento da LP apontou prevalência de $7,9 \%$ na Inglaterra e $23 \%$ na Itália. No Brasil, embora a notificação do agravo seja insatisfatória, estudos apresentam, no âmbito domiciliar, incidência de 20\%, e 23,52\% de prevalência. ${ }^{(9)}$

Frente a essa realidade, no Sistema Único de Saúde (SUS), entre as premissas exigidas, está a formação e o estímulo aos profissionais da equipe de Saúde da Família (eSF) para uma reflexão da prática que justifique ações de promoção da saúde e prevenção da LP.

Nesse contexto, o enfermeiro, como membro da eSF e líder da equipe de enfermagem, é responsável pelo gerenciamento do cuidado. Esta atuação salienta a tomada de decisão e propicia a escolha da melhor prática de cuidar a ser empregada ao paciente. ${ }^{(10)}$ Destaca-se, também, seu papel fundamental em diminuir e/ou evitar os agravos, contribuindo na promoção da qualidade de vida das pessoas e seus familiares.

Para tanto, esse profissional precisa estar guarnecido de habilidades e conhecimentos técnicos para melhor assistir, pois sua formação se fundamenta em uma estratégia das políticas de saúde e de atenção à população, em que o ensino e aprendizado são inseridos na prática do serviço, motivando para a capacidade reflexiva agentes de mudanças e produtores de conhecimentos. ${ }^{(4)}$

Em face à relevância do tema LP, à aquisição de maiores conhecimentos quanto aos efeitos deletérios e, em especial, à possibilidade de evitar o seu desenvolvimento, é objetivo deste estudo analisar, a partir da visão dos enfermeiros da Atenção Primária, as contribuições da prática educativa na prevenção da Lesão por pressão e promoção da saúde. 


\section{MÉTODO}

Trata-se de um estudo qualitativo, constituído sob a luz da Política Nacional da Promoção da Saúde e recomendações internacionais da National Pressure Ulcer Advisory Panel/European Pressure Ulcer Advisory Panel, cujo referencial metodológico utilizado foi a Pesquisa Convergente Assistencial (PCA). Essa metodologia exige do pesquisador o compromisso com a construção de um novo conhecimento, novos modos de cuidado e novas tecnologias, permitindo ao participante a renovação ou inovação da prática assistencial. ${ }^{(11)}$

Participaram do estudo 20 enfermeiros da APS (Atenção Primária a Saúde). A inclusão dos participantes se deu por ser enfermeiro da eSF do Distrito Sanitário em questão, e adotou-se como critério de exclusão, desempenhar exclusivamente funções administrativas, como Coordenador do Centro de Saúde.

A coleta de dados ocorreu em julho e agosto de 2014, no estado de Santa Catarina. Foram realizados quatro encontros com duração aproximada de 4 horas cada, todos durante a reunião técnica da própria instituição.

A PCA foi conduzida em duas etapas, a primeira, investigativa, a partir de questionário entregue no primeiro encontro à cada participante, com quatro perguntas abertas: O que você conhece sobre LP?; O que você conhece sobre os riscos de uma pessoa que está no domicílio a desenvolver a LP?; Como identifica e avalia a pessoa que está suscetível a desenvolver a LP?; e Quais ações podem prevenir o desenvolvimento da LP e promover a saúde da pessoa suscetível, a fim de evitar o agravo? Tais respostas foram compiladas e analisadas, e forneceram elementos que subsidiaram o planejamento da etapa subsequente.

A segunda etapa se constituiu da prática educativa na forma de três oficinas, tendo como tema central "Diga não à LP", a qual objetivou reflexões e a construção de um novo conhecimento com todos os envolvidos.

Cada oficina teve seu planejamento a partir da organização do espaço com disposição das cadeiras em círculo, acolhimento dos participantes, desenvolvimento da atividade central, com o compartilhamento de experiências e reflexões, e o fechamento da oficina destinado a esclarecimentos e agradecimentos.

Os trabalhos nas oficinas foram realizados em grupos, em que os participantes tiveram livre escolha de organização, possibilitando mobilidade entre eles e ampliando a probabilidade da troca de saberes. Com intuito de estimular a participação, aflorar o tema LP e enriquecer o debate nas oficinas foi empregado: leitura de textos, estudos de casos clínicos fictícios e conteúdo expositivo.

Na primeira oficina e segundo encontro, foi discutido o conceito, a classificação, a localização e os fatores de risco da LP. Na segunda oficina e terceiro encontro, foram discutidas as práticas de promoção da saúde e a prevenção da LP, pautada na escala de Braden. ${ }^{(6)}$

No quarto e último encontro, foi realizado o fechamento, solicitado feedback da intervenção educativa e a devolutiva individual, por escrito, sobre a experiência da construção e reconstrução do conhecimento acerca do tema trabalhado, a partir das seguintes questões: a primeira, destacar o que mais foi expressivo para você na construção e reconstrução do conhecimento sobre a LP, promoção da saúde e a prevenção da LP durante as oficinas; e a segunda, refletir e explicar sobre as experiências compartilhadas nas oficinas, e as mudanças na sua prática em relação à prevenção da LP e promoção da saúde.

Os dados levantados nas entrevistas foram transcritos e as oficinas gravadas. Posteriormente, foram realizadas todas as transcrições, inclusive da gravação, e a análise, a partir do referencial Teórico da Promoção da Saúde e Lesão por Pressão.

Na sequência, após diversas leituras do material transcrito, os dados semelhantes foram agrupados, originando duas categorias: diga não à Lesão por Pressão, a prática educativa na visão do enfermeiro, e as práticas desenvolvidas pelo enfermeiro na prevenção da Lesão por Pressão e promoção da saúde; e, por fim, a fase de teorização articulando as informações categorizadas com a literatura, e a de transferência dos dados. ${ }^{(11)}$ 
As atividades somente iniciaram após assinatura do Termo de Consentimento Livre e Esclarecido, sendo assegurado o anonimato com a identificação dos participantes por "E" (enfermeiro) seguido por letras.

Ressalta-se que essa pesquisa foi aprovada pelo Comitê de Ética em Pesquisa da Universidade Federal de Santa Catarina com parecer n⿳0 711.385, em 07 de julho de 2014.

\section{RESUltados}

Os resultados foram discutidos à luz do referencial teórico proposto, relacionando-os com a APS. A fim de situar o leitor, a distribuição dos participantes, segundo a caracterização sociodemográfica, é apresentada a seguir.

Desta forma, no tocante à idade, dos 20 enfermeiros do estudo, 4 (20\%) têm entre 21 e 30 anos, oito entre 31 e 40 anos, sete entre 41 e 50, sendo que 1 não respondeu. Em relação à formação profissional, 10 estavam formados entre 1 e 10 anos, 5 entre 11 e 20 anos, 2 entre 21 e 30 anos, e 3 não responderam ao questionamento. Quanto à pós-graduação, especialização, mestrado e doutorado, 19 possuíam alguma especialização, e, destes, 12 em Saúde da Família. Não houve registro de mestrado e doutorado. No que tange ao tempo de atuação na eSF, 14 apresentaram entre 1 e 10 anos, 4 entre 11 e 20 anos, e 2 não se manifestaram.

As informações foram analisadas e organizadas por afinidade, e resultaram em duas categorias: "diga não à LP, a prática educativa na visão do enfermeiro da APS", e "as práticas desenvolvidas pelo enfermeiro na prevenção da Lesão por Pressão e promoção da saúde". Tais categorias atendem ao escopo deste estudo na aquisição e aprimoramento do conhecimento sobre a LP, contribuindo para a compreensão dos profissionais da saúde, do quanto esse conhecimento é essencial para uma gestão do cuidado de Enfermagem segura e de qualidade.

\section{Diga não à LP, a prática educativa na visão do enfermeiro da APS}

Nesta categoria, os participantes apresentaram suas opiniões sobre a prática educativa enquanto estratégia de intervenção e mudança no dia a dia, tais como a identificação dos riscos de desenvolvimento do agravo; caracterizar o tipo de lesão e seus estágios; e o tratamento adequado da avaliação da LP, a partir dos conhecimentos produzidos durante a oficina.

Também pontuaram a prática educativa de forma positiva e as estratégias utilizadas nas oficinas facilitaram a compreensão e associação da teoria com a prática desses profissionais.

Permitiram avaliação da LP através de imagens (fotos). (Grupo E-L)

Facilitou o aprendizado com a construção de maquete, discussão dos casos. (Grupo E-G)

Possibilitou avaliar o contexto em que se encontra o paciente. (Grupo E-M)

Os participantes entenderam que o tema LP é merecedor de atenção pela necessidade de cuidados à pessoa que já possui ou está suscetível ao agravo. A maioria dos entrevistados revelou que a atualização dos conteúdos discutidos na prática educativa se tornou importante, pois resulta em um cuidado mais qualificado e seguro, através da supressão de práticas que já não são mais utilizadas, como uso de luvas de procedimento com água na região de calcâneo; massagem vigorosa em área de proeminência óssea e hiperemiada, com o objetivo de prevenir e promover a saúde, na busca por uma melhor qualidade de vida dessas pessoas:

Agregou mais conhecimento para [...] prevenir a LP. (Grupo E-F)

Ajudou a pensar e promover qualidade de vida ao paciente. (Grupo E-M)

Conhecer as práticas que hoje já não se faz mais, como massagem em proeminências ósseas, entre outras. (Grupo E-N) 
Os participantes destacaram que os encontros realizados de forma contínua proporcionaram abertura para a discussão e reflexão do tema nos grupos. Além disso, houve troca de saberes entre os profissionais da equipe de saúde no cuidado.

\section{A prática do enfermeiro na prevenção da LP e promoção da saúde na APS}

Os participantes relataram sobre as mudanças na assistência, entre elas, orientar a pessoa e os familiares cuidadores sobre reposicionamento no leito; aporte nutricional adequado, cuidados gerais com a pele, e outras medidas preventivas. O cuidado prestado pelo enfermeiro e a participação de outros profissionais, assim como da família nesse processo, constituindo-se em uma possibilidade de mudança:

Colocar em prática os conhecimentos com os pacientes, e ampliar estes conhecimentos para os outros profissionais. (Grupo E-A)

Nortear o atendimento ao usuário/família. (Grupo E-L)

Prestar cuidados mais eficazes, a partir da avaliação e orientação às pessoas com LP. (Grupo E-C)

No que tange ao cuidado direto à pessoa que está suscetível ao desenvolvimento da LP, o resultado aponta para ações intervenientes que buscam solucionar ou minimizar os problemas do dia a dia, com a utilização de forma sistematizada da Escala de Braden, a fim de avaliar o grau de risco para o desenvolvimento da lesão e evitar o agravo:

Prestar maior atenção no atendimento que envolve o paciente em risco de desenvolver $L P$, realizando a avaliação com a utilização da escala de Braden, que é uma novidade para muitos enfermeiros. (Grupo E-K)

Passar a ter um olhar direcionado aos cuidados de prevenção e promoção da saúde. (Grupo E-F).

Na visão dos participantes, foi apresentado como resultado para uma prática inovadora, como o reforço à autonomia e autocuidado da pessoa em seu contexto, e uma ação que prima pela Sistematização da Assistência de Enfermagem em um cuidado uniforme, consensual e científico, mas que versa pelas particularidades da realidade individual e coletiva:

Padronizar e aprimorar o cuidado de acordo com a realidade (Grupo E-D), principalmente a prescrição de cuidados de Enfermagem (Grupo E-I), e com isso melhorar a qualidade do atendimento (Grupo E-M).

\section{DISCUSSÃO}

As práticas educativas grupais preveem um caminho inovador que gera reflexões, atitudes conscientes e intencionais dos envolvidos, além da valorização e do reconhecimento profissional. ${ }^{(12)}$

Para realização das práticas educativas, é relevante trabalhar com dinâmicas diversas, incluindo o lúdico como forma de ensino/aprendizado. Na área de Enfermagem, podendo gerar opiniões, em que se privilegia uma abordagem que enfatiza as experiências e os saberes contextualizados das pessoas envolvidas, entendendo-os como processos estimuladores de mudanças individuais e coletivas. ${ }^{(13)}$

Entretanto, a equipe de enfermagem necessita de atualização e qualificação para ações educacionais, ${ }^{(4)}$ em especial, quando se trata de algo que não foge aos desafios encontrados cotidianamente pelo profissional enfermeiro no contexto da APS.

Atualizar e capacitar os profissionais de Enfermagem exigem métodos de ensino e aprendizagem dinâmicos e contínuos, e que proporcionem ao indivíduo a aquisição de conhecimentos, para que ele atinja sua capacidade profissional e desenvolvimento pessoal, de acordo com a sua realidade institucional. ${ }^{(14)}$ As dinâmicas contempladas nesta pesquisa, como estudos clínicos, leitura de textos e o lúdico, funcionaram como um elo integrador de reconhecimento do contexto vivenciado pelos enfermeiros, possibilitando agregar conhecimentos, vencer realidades angustiantes e inseguranças 
infinitas, como o reconhecimento da necessidade de realizar ações preventivas, tratamento e cuidados de enfermagem às Lesões por Pressão.

As dinâmicas vivenciadas possibilitaram reflexões entre os participantes sobre o contexto assistencial que suscitaram alternativas de soluções para os problemas cotidianos, tais como sensibilização da equipe de saúde para identificação dos fatores de risco, notificação precoce dos casos novos, vigilância e monitoramento constante, induzindo assim renovação da prática, a partir do envolvimento dos atores na pesquisa. ${ }^{(14-15)}$

Nesse raciocínio, a inserção da promoção da saúdee a prevenção de doença são necessárias para admitir mudanças no sistema de cuidado e requerem ações concretas para sua efetivação e fortalecimento, ${ }^{(16)}$ especialmente o conhecimento de ações preventivas, bem como de promoção da saúde pela equipe de Enfermagem, ${ }^{(17)}$ políticas públicas que objetivam encorajar a autonomia e segurança das pessoas com LP.

Assim, pode-se dizer que centrar esforços, dispensando uma maior atenção voltada à pessoa suscetível a desenvolver a LP, configura-se em uma ação benéfica do cuidado em Enfermagem, por ações inovadoras preventivas e de promoção da saúde, tais como a identificação dos fatores de riscos e implantação de ações efetivas de tratamento, em que o enfermeiro tem grande responsabilidade em qualquer nível de atenção.(18) Isso tendo em vista que o enfermeiro, quando cuida de pessoas com feridas, não deve compreender o seu trabalho apenas focado na execução de curativos, mas deve se tornar responsável pela integralidade da pessoa cuidada. ${ }^{(19)}$

O conhecimento sobre o cuidado ao agravo deve ser ampliado com os profissionais, de forma que seja interdisciplinar, e é considerado importante pressuposto para reorganização do processo de trabalho com práticas de promoção da saúde e prevenção de doenças, visando a uma abordagem mais integral, resolutiva $^{(18,20)}$ e definida como a base voltada à família e ao seu contexto. ${ }^{(21)}$

A análise das discussões oriundas da prática educativa também evidenciou a expansão de conceitos de cuidado, prevenção e tratamento, corroborada por um estudo que identificou que a aproximação da teoria com a prática culmina em uma visão ampliada do paciente na sua totalidade, facilitando a humanização do cuidado, ${ }^{(22-23)}$ especialmente porque a prática educativa tem papel fundamental para a prevenção e recuperação de paciente com LP.

Nessa linha de pensamento, coerente com o trazido neste estudo, a importância da utilização da escala de Braden na prática clínica, escala preditiva para o risco de LP, apresenta-se como um cuidado inovador, devendo ser rotineiramente realizada, mesmo em pessoas já com a lesão, uma vez que se pode prevenir a ocorrência em outros locais. ${ }^{(24)}$ Além disso, o enfermeiro ao cuidar de pacientes com LP deve empenhar-se nesse cuidado, direcionando esforços para a prevenção dessa condição de saúde. ${ }^{(25)}$

Os cuidados com a integridade da pele são elementos da prática do enfermeiro, tanto no cuidado de prevenção quanto de promoção da saúde, visando à melhoria da qualidade de vida das pessoas suscetíveis ao desenvolvimento da LP e seus familiares. Para tanto, uniformizar a linguagem, por meio da utilização da Escala de Braden, pode favorecer a qualificação da assistência do paciente vulnerável ao desenvolvimento da LP, ${ }^{(26-27)}$ importância elencada pelos participantes desta pesquisa como uma ação possível de execução, a partir da prática educativa.

No Brasil, iniciativas institucionais dessa natureza são pouco comuns; no entanto, essenciais, considerando a natureza multifatorial da LP e a amplitude do problema. ${ }^{(28)}$

Os limites desse estudo estão relacionados ao fato de ter sido realizado com enfermeiros da Atenção Primária de uma região específica do Sul do Brasil, limitando à generalização dos seus resultados para outras pesquisas. Recomendam-se novos estudos sobre esta temática, com intuito de compreender e estimular discussões com enfermeiros da APS sobre a prevenção da Lesão por pressão e promoção da saúde.

Ações educativas de promoção da saúde e prevenção da LP possibilitam melhor compreensão de como o enfermeiro pode aumentar a possibilidade de um cuidado inovador com expectativa de mudança do curativo para a prevenção, melhorar a qualidade da assistência da pessoa com esta condição de saúde, e, entre outros benefícios, resultar em redução nos custos para saúde pública na Atenção Primária e demais níveis de complexidade. 
Uma das funções do profissional de Enfermagem é a realização de atividades educativas voltadas à promoção da saúde e prevenção de agravos. Destaca-se, assim, a relevância da compreensão, participação, execução destas ações no seu processo de trabalho, sendo estas fundamentais para a reorientação dos atuais modelos assistenciais, bem como para o progresso científico da Enfermagem.

\section{CONSIDERAÇÕES FINAIS}

Considerando o objetivo do estudo de analisar, a partir da visão dos enfermeiros da Atenção Primária, as contribuições da prática educativa para a promoção da saúde, observou-se que as mesmas constituemse como linhas norteadoras essenciais para formação de produtos ou ações que necessitam ser, cada vez mais, implementadas por profissionais de saúde, a fim de proporcionar um cuidado de saúde mais dinâmico e inovador.

Durante o estudo, constatou-se a necessidade de cursos de formação e atualizações sobre a escala de Braden, ferramenta contributiva para o profissional de saúde, e por consequência para o cuidado com LP. Isso porque facilita o cuidado e orienta de forma consensual, científica, respeitando as particularidades de cada indivíduo.

Sendo assim, práticas educativas permitiram que os profissionais de Enfermagem aprendessem, de forma dialógica, com consciência crítica proporcionar o desenvolvimento de habilidades para cuidar de pessoas com LP, com novos conhecimentos em ações preventivas e diagnóstico precoce, de forma a impactar positivamente na sua qualidade de vida. Isso proporciona segurança ao profissional de saúde, e dessa forma o torna mediador de conhecimentos para LP, consequentemente inovando sua prática.

Dessa forma, o resultado das oficinas mostrou enfermeiros interessados em discutir o assunto, o que é importante por resultar em um cuidado qualificado e dinâmico. Isso possibilita perspectivas de mudanças na prática diária dos profissionais, originando assim um cuidado inovador, que gera prevenção e promoção da saúde na Atenção Primária. Contudo, esta pesquisa identificou a necessidade de mais encontros contínuos com conteúdos sequenciais, para abertura de espaço para o raciocínio lógico, com discussão e reflexão de grupo.

\section{- REFERÊNCIAS}

1. Bonetti A, da Silva DGV, Trentini M. Método da pesquisa convergente assistencial em um estudo com pessoas com doença arterial coronariana. Esc. Anna Nery. [Internet] 2013;17(1) [acesso em 10 maio 2017. Disponível: http://dx.doi. org/10.1590/S1414-81452013000100025.

2. Kawata LS, Mishima SM, Chirelli Mara Quaglio, Pereira MJB, Matumoto S, Fortuna CM. Os desempenhos da enfermeira na saúde da família: construindo competência para o cuidado. Texto contexto - enferm. [Internet] 2013;22(4) [acesso em 29 maio 2017]. Disponível: http://dx.doi.org/10.1590/S0104-07072013000400012.

3. Ascari RA, Veloso J, da Silva OM, Kessler M, Jacoby AM, Schwaab G.Úlcera por pressão: um desafio para a enfermagem. Brazilian Journal of Surgery and Clinical Research - BJSCR. [Internet] 2014;6(1) [acesso em 02 maio 2017 ]. Disponível: http://www.mastereditora.com.br/bjscr.

4. Medina MG, Aquino R, Vilasbôas ALQ, Mota E, Pinto Júnior EP, da Luz LA, et al. Promoção da saúde e prevenção de doenças crônicas: o que fazem as equipes de Saúde da Família?. Saúde debate. [Internet] 2014;38(n. esp) [acesso em 29 maio 2017]. Disponível: http://dx.doi.org/10.5935/0103-1104.2014S006.

5. Both JE, Leite MT, Hildebrandt LM, Beuter M, Muller LA, Linck CL. Qualificação da equipe de enfermagem mediante pesquisa convergente assistencial: contribuições ao cuidado do idoso hospitalizado. Esc. Anna Nery. [Internet] 2014;18(3) [acesso em 29 maio 2017]. Disponível: http://dx.doi.org/10.5935/1414-8145.20140069. 
6. National Pressure Ulcer Advisory Panel (NPUAP), European Pressure Ulcer Advisory Panel (EPUAP) and Pan Pacific Pressure Injury Alliance (PPPIA). Prevention and Treatment of Pressure Ulcers: Quick Reference Guide. Emily Haesler (Ed.). [Internet] Australia: NPUAP/EPUAP/PPPIA; 2014 [acesso em 2 maio 2017]. Disponível: http://www.npuap.org/wpcontent/uploads/2014/08/Updated-10-16-14-Quick-Reference-Guide-DIGITAL-NPUAP-EPUAP-PPPIA-16Oct2014. pdf.

7. Cameron R, Rodgers A, Welsh L, McGown K. Developing eLearning for pressure ulcer prevention and management. J Nurs. [Internet] 2014;23(Suppl 15) [acesso em 29 maio 2017]. Disponível: http://dx.doi.org/10.12968/bjon.2014.23. Sup15.s16.

8. Simão CMF, Caliri MHL, dos Santos CB. Concordância entre enfermeiros quanto ao risco dos pacientes para úlcera por pressão. Acta paul. enferm. [Internet] 2015;26(1) [acesso em 29 de maio 2017]. Disponível: http://dx.doi. org/10.1590/S0103-21002013000100006.

9. Freitas JPC, Alberti LR. Aplicação da Escala de Braden em domicílio: incidência e fatores associados à úlcera por pressão. Acta paul. enferm. [Internet] 2013;26(6) [acesso em 05 jun 2017]. Disponível: http://dx.doi.org/10.1590/S010321002013000600002

10. Bezerra SMG, Luz MHBA, Andrade EMLR, de Araújo TME, Teles JBM, Caliri MHL. Prevalência, fatores associados e classificação de úlcera por pressão em pacientes com imobilidade prolongada assistidos na estratégia saúde da família. Rev. Estima. [Internet] 2014;12(2) [acesso em 11 jun 2017]. Disponível: http://www.revistaestima.com.br/index.php/ estima/article/view/95.

11. Alvim NAT. Pesquisa Convergente Assistencial Enfermagem - Possibilidades para inovações tecnológicas. Esc Anna Nery. [Internet] 2017;21(2) [acesso em 30 jan 2017]. Disponível: http://dx.doi.org/10.5935/1414-8145.20170041.

12. Pivoto FL, Lunardi Filho WD, Santos SSC, Lunardi VL. Convergente assistencial research: an integrative review of scientific nursing production. Texto contexto - enferm. [Internet] 2013;22(3) [acesso em 30 jan 2017]. Disponível: http:// dx.doi.org/10.1590/S0104-07072013000300034.

13. Mendes R, Fernandez JCA, Sacardo DP. Promoção da saúde e participação: abordagens e indagações. Saúde debate. [Internet] 2016;40(108) [acesso em 29 mai 2017]. Disponível: http://dx.doi.org/10.1590/0103-1104-20161080016.

14. Mallmann DG, Galindo NNM, Sousa JC, de Vasconcelos EMR. Educação em saúde como principal alternativa para promover a saúde do idoso. Ciênc. saúde coletiva. [Internet] 2015; 20(6):1763-1772 [acesso em 31 mai 2017]. Disponível: http://dx.doi.org/10.1590/1413-81232015206.02382014.

15. Vieira CPB, de Oliveira EWF, Ribeiro MGC, Luz MHBA, Araújo ODA, Pereira AFM. Ações preventivas em úlceras por pressão realizadas por enfermeiros na atenção básica. [Internet] 2016;8(2) [acesso em 05 jun 2017]. Disponível: http://dx.doi.org/10.9789/2175-5361.2016.v8i2.4447-4459.

16. de Souza RCR, Soares E. Implementando inovações em saúde com base na pesquisa convergente assistencial. J. Res.: Fundam. Care. [Internet] 2013;5(4) [acesso em 13 mar 2016]. Disponível: http://bases.bireme.br/cgi-bin/wxislind. exe/iah/online/?IsisScript=iah/iah.xis\&src=google\&base=BDENF\&lang=p\&nextAction=Ink\&exprSearch=24974\&indexSearch=ID.

17. Talens BF. Formación y prevención en úlceras por presión: prevalencia en el Hospital General de Elche / Training and pressure ulcer prevention: prevalence in the General Hospital of Elche. Gerokomos. [Internet] 2016:27(1) [acesso em 31 mai 2017. Disponível: http://scielo.isciii.es/scielo.php?script=sci_arttext\&pid=S1134-928X2016000100008.

18. Salci MA, Maceno P, Rozza SG, Silva DMGV, Boehs AE, Heidemann ITSB. Educação em saúde e suas perspectivas teóricas: algumas reflexões. Texto contexto - enferm. [Internet] 2013;22(1) [acesso em 31 mai 2017]. Disponível: http:// dx.doi.org/10.1590/S0104-07072013000100027.

19. dos Santos CT, Oliveira MC, Pereira AGS, Suzuki LM, Lucena AF. Indicador de qualidade assistencial úlcera por pressão: análise de prontuário e de notificação de incidente. Rev. Gaúcha Enferm. [Internet] 2013;34(1) [acesso em 13 mar 2016]. Disponível: http://hdl.handle.net/10183/85388. 
20. da Silva DAJ, Tavares MFL. Ação intersetorial: potencialidades e dificuldades do trabalho em equipes da Estratégia Saúde da Família na cidade do Rio de Janeiro. Saúde debate. [Internet] 2016;40(111) [acesso em 29 mai 2017]. Disponível: http://dx.doi.org/10.1590/0103-1104201611115.

21. de Souza JM, Tholl AD, Córdova FP, Heidemann ITSB, Boehs AE, Nitschke RG. Aplicabilidade prática do empowerment nas estratégias de promoção da saúde. Ciênc. saúde coletiva. [Internet] 2014;19(7) [acesso em 31 mai 2017]. Disponível: http://dx.doi.org/10.1590/1413-81232014197.10272013.

22. Heidemann ITSB, Wosny AM, Boehs AE. Promoção da Saúde na Atenção Básica: estudo baseado no método de Paulo Freire. Ciênc. saúde coletiva. [Internet] 2014;19(8) [acesso em 12 fev 2016]. Disponível: http://dx.doi. org/10.1590/1413-81232014198.11342013

23. da Silva AL, da Silva LF, Souza IEO, Moreira RVO. Mulher cardiopata com úlcera por pressão: reflexão fenomenológica sobre um modelo de cuidado clínico de conforto. Esc. Anna Nery. [Internet] 2013;17(1) [acesso em 13 mar 2016]. Disponível: http://dx.doi.org/10.1590/S1414-81452013000100023.

24. Hilda SS, Radunz V, da Rosa LM. O cuidar de si para promoção da saúde: os cuidados da enfermagem / Caring for self to promote health: transforming nursing care abstract. Ciênc. cuid. saúde. [Internet] 2015;14(1) [acesso em 12 mai 2017]. Disponível: http://pesquisa.bvsalud.org/portal/resource/pt/bde-29596.

25. Borghardt AT, do Prado TN, Bicudo SDS, Castro DS, Bringuente MEO. Pressure ulcers in critically ill patients: incidence and associated factors. Rev. Bras. Enferm. [Internet] 2016; 69(3) [acesso em 12 mai 2017]. Disponível: http:// dx.doi.org/10.1590/0034-7167.2016690307i.

26. Newham R, Hudgel L. Pressure-ulcer management and prevention in acute and primary care. Br J Nurs. [Internet] 2015;4(6) [acesso em 05 mar 2016]. Disponível: http://dx.doi.org/10.12968/bjon.2015.24.Sup6.S4.

27. Acendra JJ, Mercado MH, Montes L, Pardo RMA. Cumplimiento de las actividades de enfermería en la prevención de úlceras por presión. Rev Cubana Enferm. [Internet] 2016;32(2) [acesso em 05 jun 2017]. Disponível: http://scielo.sld. cu/scielo.php?script=sci_arttext\&pid=S0864-03192016000200002.

28. Pereira AGS, dos Santos CT, Menegon DB, Mello B, Azambuja F, Lucena AF. Mapeamento de cuidados de enfermagem com a NIC para pacientes em risco de úlcera por pressão. Rev. esc. enferm. USP. [Internet] 2014; 48(3) [acesso em 01 mar 2016]. Disponível: http://dx.doi.org/10.1590/S0080-623420140000300010. 